\title{
Transvaginal ligation of descending branch of uterine artery: could be the first surgical attempt to control post-partum haemorrhage?
}

\author{
Marco Mitidieri $^{1 *}$, Elisa Picardo ${ }^{1}$, Pierluigi Tondo $^{2}$, Chiara Benedetto ${ }^{2}$, Saverio Danese ${ }^{1}$
}

${ }^{1}$ Città della Salute e della Scienza di Torino, Ginecologia e Ostetrica SC4, ${ }^{2} 1 \mathrm{U}$, Torino, Italy

Received: 03 February 2021

Accepted: 25 March 2021

\section{*Correspondence:}

Dr. Marco Mitidieri,

E-mail: mitidieri.m@gmail.com

Copyright: ( $\odot$ the author(s), publisher and licensee Medip Academy. This is an open-access article distributed under the terms of the Creative Commons Attribution Non-Commercial License, which permits unrestricted non-commercial use, distribution, and reproduction in any medium, provided the original work is properly cited.

\begin{abstract}
Post-partum haemorrhage is the major cause of maternal death worldwide. This severe clinical condition can cause also physical morbidity and psychological distress (anemia, coagulopathy, blood transfusion, anterior pituitary ischemia with delay or failure of lactation, myocardial ischemia, postpartum depression). To date several efforts have been made to prevent and treat this severe condition mainly in three ways: medical, surgical, and interventional radiology even in combination. The surgical approach, needs the knowledge of anatomy of vascular distribution of the uterus. According to Palacios-Jaraquemada the feeding vessels of the body of the uterus is defined S1 area and the lower segment, uterine cervix and upper part of the vagina, S2 area. We report three cases in which the ligation of the descending branch of uterine artery (S2 area) helped the surgeon in the treatment of severe primary post-partum haemorrhage causing a significant reduction in blood loss.
\end{abstract}

Keywords: Post-partum haemorrhage, Ligation, Uterine artery

\section{INTRODUCTION}

Post-partum haemorrhage is the major cause of maternal death worldwide. This severe clinical condition can cause also physical morbidity and psychological distress (anemia, coagulopathy, blood transfusion, anterior pituitary ischemia with delay or failure of lactation, myocardial ischemia, postpartum depression). The American college of obstetricians and gynecologists defines early postpartum haemorrhage as at least 1,000 $\mathrm{ml}$ total blood loss or loss of blood coinciding with signs and symptoms of hypovolemia within 24 hours after delivery of the fetus or intrapartum loss. ${ }^{1}$ To date several efforts have been made to prevent and treat this severe condition mainly in three ways: medical, surgical, and interventional radiology even in combination. ${ }^{2}$ The surgical approach, needs the knowledge of anatomy of vascular distribution of the uterus. According to PalaciosJaraquemada the feeding vessels of the body of the uterus is defined $S 1$ area and the lower segment, uterine cervix and upper part of the vagina, S2 area. ${ }^{3}$

\section{CASE SERIES}

We report three cases in which the ligation of the descending branch of uterine artery ( $\mathrm{S} 2$ area) helped the surgeon in the treatment of severe primary post-partum haemorrhage causing a significant reduction in blood loss. In this procedure we pull down the cervix with two sponge forceps contralateral to the site of the ligation, then a polyglactin 910n 1 suture placed at 3 and 9 o'clock $1 \mathrm{~cm}$ below the cervico-vaginal junction (Figure 1). ${ }^{4}$

\section{Case 1}

A 31-year-old $1^{\text {st }}$ gravida at 35 weeks of gestational age delivered a $2300 \mathrm{~g}$ baby after medical induction of labour due to preterm rupture of the membranes. The heavy uterine bleeding (estimated $3000 \mathrm{ml}$ ) started after a complete third stage of labour. We administered medical treatment (oxytocin, methylergometrine and sulprostone), then considering the persistent bleeding we performed a curettage of the uterus and in absence of vaginal and 
cervical tears we tied the cervical knots before insert the Bakry balloon for compression. The emergency was controlled, three units of blood were transfused.

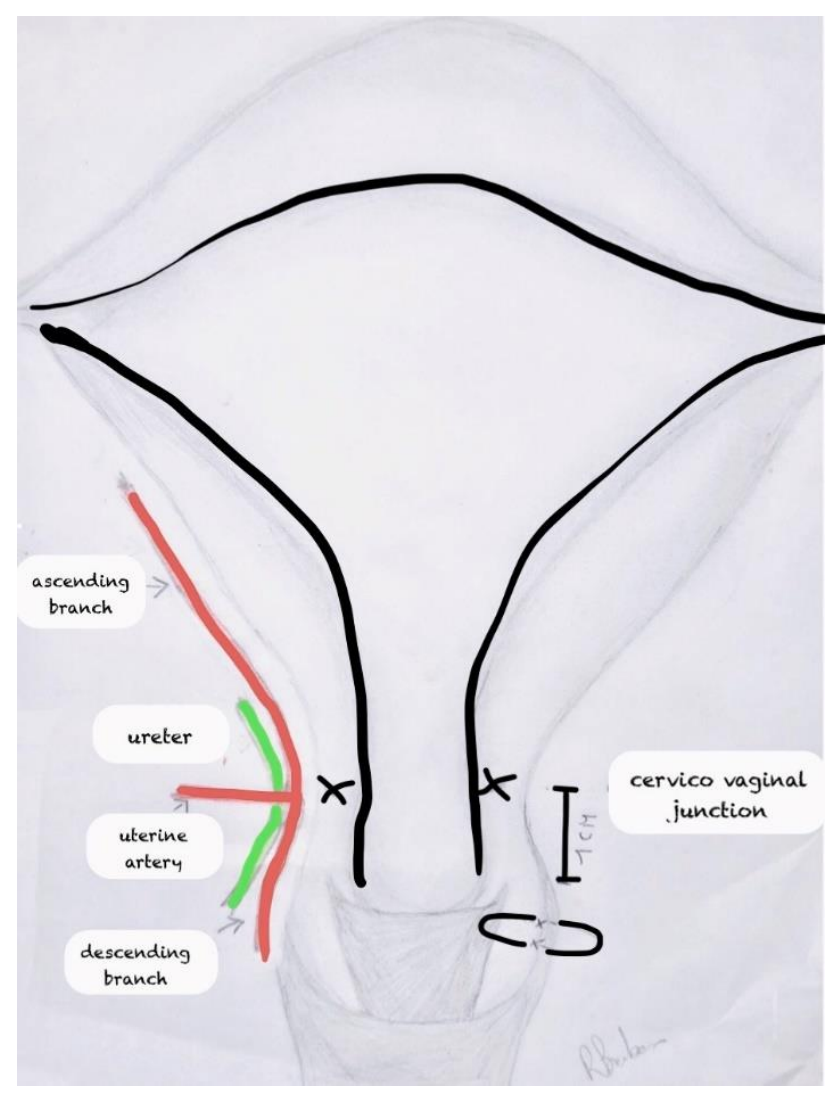

Figure 1: On the left side uterine artery crosses over the ureter and divides into ascending and descending branch. On the right side the suture is placed in safety, $1 \mathrm{~cm}$ below the cervico-vaginal junction.

\section{Case 2}

A 22-year-old $1^{\text {st }}$ gravida at 41 weeks of gestational age after a spontaneous delivery of $3800 \mathrm{~g}$ baby suffered of retained placenta. We proceed with manual removal of the placenta, curettage and medical therapy. Due to the continuous bleeding $(3000 \mathrm{ml})$ and in absence of causes of trauma we put the cervical knots at 3 and 9 o'clock to start control the blood loss before positioning Bakry balloon. The bleeding was controlled, the patient needs the transfusion of two blood unit.

\section{Case 3}

A 36-year-old $1^{\text {st }}$ gravida at 40 weeks of gestational age after a spontaneous delivery of $3270 \mathrm{gm}$ baby experienced retained placenta. The manual removal of the placenta, curettage and medical therapy proved insufficient to control the heavy bleeding $(2000 \mathrm{ml})$. We performed the ligation of the descending branch of the uterine artery according to the procedure described and then positioned the Bakry balloon.
We transfused two blood unit. For all cases, the discharge was the $5^{\text {th }}$ day, at $30^{\text {th }}$ day follow up visit the pelvic examination was regular and breastfeeding maintained.

After those experiences our hypothesis is that devascularize the S2 area before the other procedures could be safe and simple, reduce the blood loss, the need of transfusions and the days of hospital stay.

\section{DISCUSSION}

To perform a successful ligation of descending branch of uterine artery it is important to know the origin, distribution and anastomosis of the genital arterial pedicles, in particular the topographic area, called S2, that includes the lower segment, uterine cervix and upper part of the vagina. ${ }^{5}$ In rare cases the cervical artery could have an unusual path and the resulting bleeding can only be stop by detecting and ligating the aberrant vessel. ${ }^{6}$ The ligation of descending branch of uterine artery to control intractable genital tract bleeding as a fertility sparing approach is dealt in few case reports. Moiranghtem described this procedure to control vaginal bleeding occurred at the time of voluntary termination of pregnancy as well as in case of primary post-partum haemorrhage following lower segment caesarean section for placenta previa. ${ }^{4}$ Moreover, the ligature of descending branch of uterine artery proved effective also in a case of secondary post-partum haemorrhage. ${ }^{7}$ Lastly, Ratten described the treatment of a cervical pregnancy with this technique in order to avoid a demolition approach in a fertile woman. ${ }^{8}$

\section{CONCLUSION}

In a clinical scenario with low evidences derived from RCTs the ligation of descending branch of uterine arteries before other procedures (surgical, compressive or endovascular) could be investigated due to the simpleness and the low probability of side effects.

\section{ACKNOWLEDGMENTS}

Author would like to thanks to Rosanna Barbaro for the illustration (Figure 1).

\section{Funding: No funding sources Conflict of interest: None declared Ethical approval: Not required}

\section{REFERENCES}

1. American College of Obstetricians and Gynecologists. Obstetric data definitions (version 1.0). Revitalize. https://www.acog.org//media/Departments/Patient-Safety-and-QualityImprovement/2014reVITALize

ObstetricDataDefinitionsV10.pdf. Accessed October 2,2016 . 
2. Kellie FJ, Wandabwa JN, Mousa HA, Weeks AD. Mechanical and surgical interventions for treating primary postpartum haemorrhage. Cochrane Database Systematic Rev. 2020;7:CD013663

3. Palacios-Jaraquemada JM. Efficacy of surgical techniques to control obstetric hemorrhage: analysis of 539 cases. Acta Obstet Gynecol Scand. 2011;90(9):1036-42.

4. Moirangthem RS. Bilateral vaginal ligation of descending branch of the uterine artery in the management of heavy genital tract bleeding. J Obstet Gynecol India. 2005;55(6):2005.

5. JPalacios-Jaraquemada JM. Lower uterine blood supply: extrauterine anastomotic system and its application in surgical devascularization techniques. Acta Obstetricia et Gynecologica. 2007;86:228234.

6. Chordia SKS. Management of a bleeding aberrant cervical artery following vacuum suction termination. Case report. Bri J Obstet Gynaecol. 1988;95:411-3.

7. Chandra R, Singh S. Vaginal ligation of descending branch of uterine artery in the management of massive secondary post-partum haemorrhage. Int $\mathbf{J}$ Reprod Contracept Obstet Gynecol. 2019;8:2937-9.

8. Ratten GJ. Cervical pregnancy treated by ligation of the descending branch of the uterine arteries. Case report, Bri J Obstetr Gvnaecol. 1983;90:367-71.

Cite this article as: Mitidieri M, Picardo E, Tondo $\mathrm{P}$, Benedetto C, Danese S. Transvaginal ligation of descending branch of uterine artery: could be the first surgical attempt to control post-partum haemorrhage? Int J Reprod Contracept Obstet Gynecol 2021;10:2042-4. 